\title{
Quality assessment for mobile media-enriched services: impact of video lengths
}

\author{
Jose Oscar Fajardo*, lanire Taboada and Fidel Liberal
}

\author{
* Correspondence: joseoscar. \\ fajardo@ehu.es \\ Department of Electronics and \\ Telecommunications, University of \\ the Basque Country, Bilbao, Spain
}

\begin{abstract}
The inclusion of multimedia content in different web-based services has increased significantly. Through an extensive subjective testing campaign, we analyse the quality of experience concerning video transmissions associated to these types of services when accessed from mobile devices and mobile Internet connections. Contrary to traditional normalised quality assessment studies, we point out the service context as a key aspect in quality assessments. Specifically, we analyse the impact of the duration of the test material on quality assessments. We find out that tolerance to visual degradations is higher in the specific context of use compared to when using standardised methodologies for quality assessment, which has a significant impact in terms of commercial service acceptability.
\end{abstract}

\section{Quality in web-based media-enriched services}

The explosion of multimedia content on the Internet has attracted a number of commercial players. New media-enriched services are constantly being deployed on the web, such as mass media, online advertising in web pages, user interaction in social networks, user-generated content sharing portals, etc. At the same time, the segment of users who access these types of services through mobile connections is growing significantly.

The quality of experience (QoE) as expressed by end users for these services is of paramount importance for service and network engineers, since it eventually influences customers' willingness to use the service. Although quality in video transmissions has been thoroughly studied in recent years, it has been commonly associated to visual quality [1,2] instead of addressing the problem from a pure service standpoint. Ibarrola proposes in [3] a general model for the management of quality of service (QoS) based on ITU-T E.802, where the concept of service is linked to the service context since it modifies both users' expectations and perceptions. Less work has been devoted to the study of multimedia services in their context of use. In [4] authors overview several studies concerning the key influence of the context in service quality evaluations: the upper and lower thresholds of satisfaction seem to be attenuated in the specific context of use. In [5], users are requested to watch full length movies with different types of degradations along the video track. Results show that the perceived visual quality is considerably different when compared to the traditional short duration tests. Additionally, users show different tolerance levels when movies are played at TV or PC screens, which points out that users' expectations play a major role in service evaluations. 


\section{User experimentation for mobile context of use}

This paper illustrates a series of experiments aimed at analyzing the QoE for different media-enriched web-based services in a mobile context. An extensive subjective testing campaign was carried out to gauge the satisfaction as expressed by end users. 20 subjects participated in the tests ( 13 male, 7 female) with ages ranging from 21 to 44 years old (average at 28). Context of use was explored using (1) video property and length (2) mobile access and (3) the methodology for assessing user experience. Users were asked to evaluate the impact of similar visual impairments in two contexts: (1) using common test video sequences and including typical wireless degradations, and (2) including similar loss conditions into video sequences extracted from considered online services.

Video lengths were inferred from the analysis of different online sources, namely BBC news mobile, YouTube Mobile and Facebook. The duration of clips ranged from $20 \mathrm{~s}$ to $600 \mathrm{~s}$, with average values between $94 \mathrm{~s}$ and $197 \mathrm{~s}$. In [6] authors found average duration of videos hosted in Daum (popular service for user-generated content in Korea) from $30 \mathrm{~s}$ for advertisements to $203 \mathrm{~s}$ for music videos. Concerning viewing conditions, all sequences were displayed in a mobile handset (screen size of 2.8 inches, resolution of $320^{*} 240$ pixels) instead of a normalised LCD display, and users were asked to hold the handset on their hands with a free viewing distance (commonly 6-8 times the height of the display). For quality assessments, we decided not to use the recommended continuous assessment method for long video sequences, since quality evaluation tasks may distort the results from a service perspective. Instead, absolute category rating (ACR) was used at the end of each sequence. In addition to quantitative assessments, qualitative evaluations were considered allowing users to add comments and to stop the play out if quality was perceived as unacceptable.

\section{Short video sequences}

In the initial phase, users were asked to evaluate the visual quality of impaired short video sequences. Common test video sequences in traditional visual quality studies have been selected, namely "football", "stefan", "carphone" and "suzie". According to the classification in [7], these four video sequences provide a good sample for the different quadrants in the spatio-temporal complexity grid in order to take into account different content types. Video sequences were degraded based on the wireless error model therein presented for mobile Internet connections. Figure 1 illustrates the set of impaired video sequences selected for the aims of this paper and the results from the quality evaluations. For each considered video clip, we show the evolution of the resulting structural similarity index (SSIM) respect to the original sequence, as a means for estimating the severity of the impairments from an objective quality metric standpoint. At the rightmost subplots, we present the results obtained from the subjective tests in terms of mean opinion score (MOS). All the obtained quality assessments are quite poor and are considered unacceptable for a commercial service.

\section{Long video sequences}

During the second phase, two different types of videos were considered in order to capture different spatio-temporal characteristics. We first analyse the results with a severe degradation of $6 \mathrm{~s}$ in the middle of a "high complexity"-"high motion" video sequence, corresponding to an "nba top ten plays of the week" clip of $100 \mathrm{~s}$. Figure 2 illustrates the degradation pattern and the subjective assessments as provided by users. 

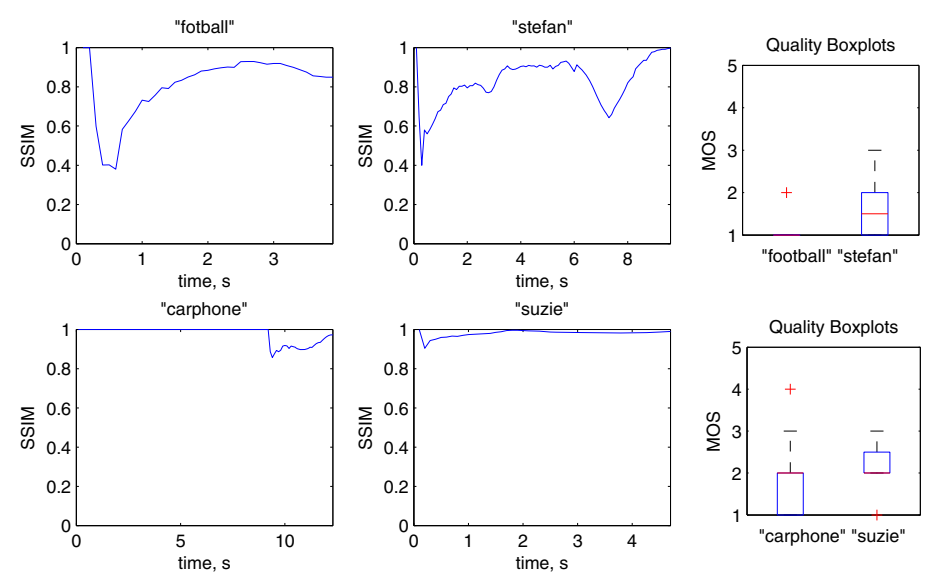

Figure 1 Evolution of SSIM and quality scores for impaired short sequences. Left top (LT) and central top (CT): "high complexity"-"high motion" video sequences; left bottom (LB): "low complexity"-"high motion" video sequence;:central bottom (CB): "low complexity"-"low motion" video sequence; right top (RT) and right bottom (RB): boxplots of quality assessments.

In general, the quality evaluations are considerably higher compared to short video sequences. In terms of MOS the video clip scores 3.31, which can be considered in the lower range of acceptability for commercial services. However, the variability of quality assessments is substantial compared to short clips with the same group of individuals. These results indicate that user segmentation shall be necessary for an accurate inclusion of users' expectations, as described in [3]. Taking into account the qualitative evaluations provided by users, some of them state that "Very good quality, except a severe degradation in the middle" or "If repeated, it would be unacceptable". As a result, one isolated severe impairment in such service contexts is not enough to abort the session, but the provider should maximise the quality policies to assure an accurate network performance for rest of session lifetime.

The second experiment introduces diverse degradations in a "low complexity" clip, namely a "talkshow" sketch of 120 s. Figure 3 illustrates the different levels of degradations used in the subjective tests. In the left-top plot three different sequences are illustrated, ranging from several light $2 \mathrm{~s}$ degradations to one severe $10 \mathrm{~s}$ degradation. The associated boxplot (right top) gathers the statistics for the aggregated quality
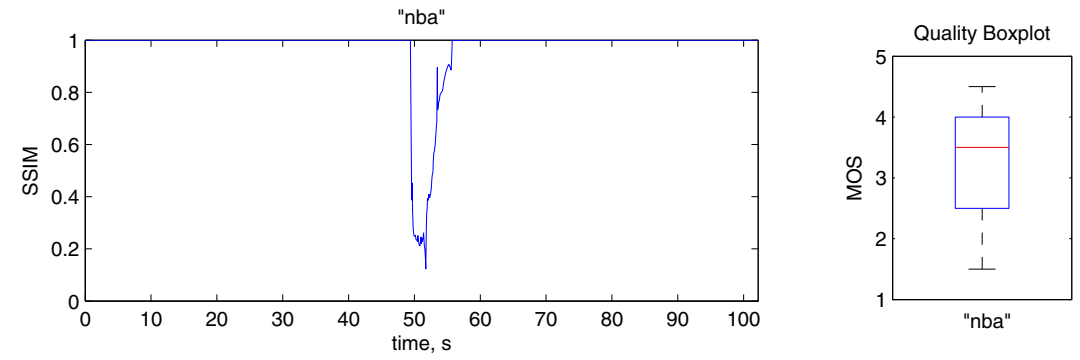

Figure 2 Evolution of SSIM and quality scores for impaired long sequence - high spatial complexity 

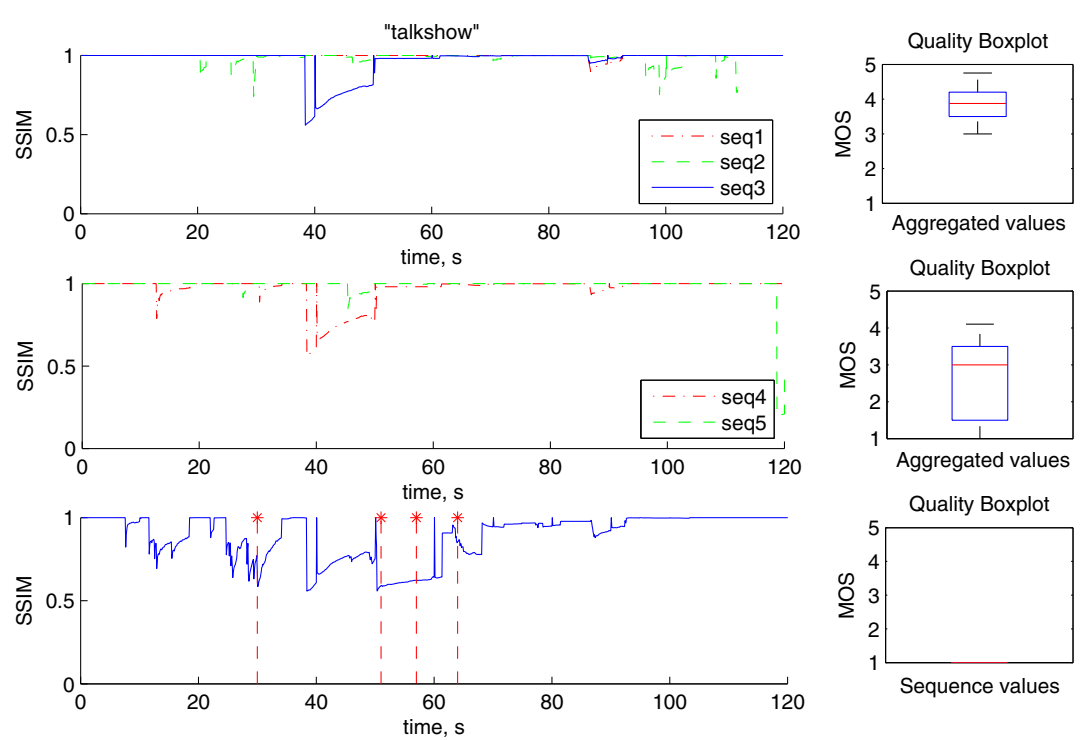

Figure 3 Evolution of SSIM and quality scores for impaired long sequences - low spatial complexity.

assessments. Obtained results are considerably different to short video clips: the visual quality is perceived from fair to excellent with no comments about acceptability. Hence, results from traditional quality studies are not directly applicable to these media-enriched services. Central plots illustrate the evolution of image impairments with additional degradations (left) and the associated subjective quality scores (right). Although obtained quality scores are lower, the experienced quality is indeed higher compared to short clips with less severe degradations. Two individuals stated that "I would stop the video if degradations persist". Thus, once again the variability of quality scores may advise towards user segmentation for an optimal management. Finally, the left bottom plot shows the SSIM for a highly degraded video sequence. $80 \%$ of people who evaluated the sequence stopped the reproduction before the end of the transmission, and the remaining subjects provided the lowest quality score as well. However, as illustrated with vertical dotted lines, the acceptability threshold is variable and difficult to gauge with the limited set of tests.

\section{Conclusion}

We show the QoE results concerning video transmissions associated to a series of media-enriched web services in a mobile context of use. We revisit the relevance of traditional visual quality assessment studies from a service deployment standpoint. From an extensive subjective testing campaign we find out that users' tolerance to visual degradations is quite higher when video sequences of 100-120 s are considered, as typical values for the considered services. This effect should be taken into account when deploying mobile media services or proposing real-time adaptation actions. As well, the variability of users' assessments indicates that user segmentation could be a good input for defining these kinds of management strategies. 


\section{References}

1. Winkler S, Mohandas P (2008) The evolution of video quality measurement: From PSNR to hybrid metrics. IEEE T Broadcast 54:660-668

2. Lin W, Jay-Kuo CC (2011) Perceptual visual quality metrics: A survey. J Vis Commun Image R 22:297-312. doi:10.1016/j. jvcir.2011.01.005

3. Ibarrola E, Liberall F, Ferro A, Xiao J (2010) Quality of service management for ISPs: A model and implementation methodology based on the ITU-T recommendation E.802 framework. IEEE Commun Mag 48:146-153

4. Jumisko-Pyykkö S, Utriainen T (2010) A Hybrid Method for Quality Evaluation in the Context of Use for Mobile (3D) Television. Multimed Tools Appl 55:185-225

5. Staelens N, Moens S, van den Broeck W, Mariën I, Vermuelen B, Lambert P, van de Walle R, Demeester P (2010) Assessing quality of experience of IPTV and video on demand services in real-life environments. IEEE T Broadcast 56:458-466

6. Cha M, Kwak H, Rodriguez P, Ahn YY, Moon S (2009) Analyzing the Video Popularity Characteristics of Large-Scale User Generated Content Systems. IEEE/ACM T Network 17:1357-1370

7. Khan A, Sun L, Ifeachor E, Fajardo JO, Liberal F (2010) Video quality prediction models based on video content dynamics for H.264 video over UMTS networks. Int J Digital Multimedia Broadcasting 2010:608138

doi:10.1186/2192-1121-1-2

Cite this article as: Fajardo et al:: Quality assessment for mobile media-enriched services: impact of video lengths. Communications in Mobile Computing 2012 1:2.

\section{Submit your manuscript to a SpringerOpen ${ }^{\circ}$} journal and benefit from:

- Convenient online submission

- Rigorous peer review

- Immediate publication on acceptance

- Open access: articles freely available online

- High visibility within the field

- Retaining the copyright to your article

Submit your next manuscript at $\boldsymbol{s p r i n g e r o p e n . c o m ~}$ 